\title{
Aching face and hand: the interoceptive attentiveness and social context in relation to empathy for pain
}

\author{
Michela Balconi ${ }^{1,2}$, Laura Angioletti ${ }^{1,2, *}$ \\ ${ }^{1}$ International Research Center for Cognitive Applied Neuroscience (IrcCAN), Faculty of Psychology, Università Cattolica del Sacro Cuore, 20123 Milan, Italy \\ ${ }^{2}$ Research Unit in Affective and Social Neuroscience, Department of Psychology, Università Cattolica del Sacro Cuore, 20123 Milan, Italy \\ *Correspondence: laura.angioletti1@unicatt.it (Laura Angioletti)
}

DOI:10.31083/j.jin2101034

This is an open access article under the CC BY 4.0 license (https://creativecommons.org/licenses/by/4.0/).

Submitted: 29 April 2021 Revised: 10 May 2021 Accepted: 30 July 2021 Published: 28 January 2022

This research explored how the manipulation of interoceptive attentiveness (IA) can influence the frontal (dorsolateral prefrontal cortex (DLPFC) and somatosensory cortices) activity associated with the emotional regulation and sensory response of observing pain in others. 20 individuals were asked to observe face versus hand, painful/non-painful stimuli in an individual versus social condition while brain hemodynamic response (oxygenated $\left(\mathrm{O}_{2} \mathrm{Hb}\right.$ ) and deoxygenated hemoglobin ( $\mathrm{HHb}$ ) components) was measured via functional Near-Infrared Spectroscopy (fNIRS). Images represented either a single person (individual condition) or two persons in social interaction (social condition) both for the pain and body part set of stimuli. The participants were split into experimental (EXP) and control (CNT) groups, with the EXP explicitly required to concentrate on its interoceptive correlates while observing the stimuli. Quantitative statistical analyses were applied to both oxy- and deoxy- $\mathrm{Hb}$ data. Firstly, significantly higher brain responsiveness was detected for pain in comparison to no-pain stimuli in the individual condition. Secondly, a left/right hemispheric lateralization was found for the individual and social condition, respectively, in both groups. Besides, both groups showed higher DLPFC activation for face stimuli presented in the individual condition compared to hand stimuli in the social condition. However, face stimuli activation prevailed for the EXP group, suggesting the IA phenomenon has certain features, namely it manifests itself in the individual condition and for pain stimuli. We can conclude that IA promoted the recruitment of internal adaptive regulatory strategies by engaging both DLPFC and somatosensory regions towards emotionally relevant stimuli.

\section{Keywords}

Interoceptive attentiveness; Pain; Face; Hand; Empathy; fNIRS

\section{Introduction}

Can the manipulation of interoception affect the mirroring empathic effect of observing another person receiving a painful stimulation? Which effects does the attention to internal states produce at the cortical level? The term "interoception" refers to the perception of the afferent signals on the bodily status and homeostasis, primarily associated with the physiological condition of the body. Nevertheless, recently the construct definition has been expanded and encompass the brain correlates and mental representations of the inter- nal bodily changes, that is cognitive, and emotional processes with varying degrees of awareness, spanning from unconscious to conscious levels [1-3]. Interoception consists of multiple different dimensions ranging from the brain-andbody to the cognition level: interoceptive sensitivity (IS) is a first dimension routinely measured as a person's capacity to sense and properly report one's heartbeats at rest; while the dimension of interoceptive awareness (IAw) corresponds to subject's confidence in his/her own behavioral performance controlled on the basis of his/her accuracy performance in Heart Beat Detection task [3, 4]. Previous studies conceived those interoception dimensions (i.e., IS and IAw) as a relatively stable trait that might alter both the subjective experience of emotion and the capacity to differentiate "self" from "other" in the empathic resonance of action [5]. On the other hand, experimentation showed that cortical representations of interoceptive attention can be modified by specific training [6]. Interoceptive attentiveness (IA), that is "the focused attention to a particular interoceptive signal for a given time interval" [7], can be included in another dimension of interoception that is a higher-level dimension (going beyond metacognition, namely executive attribution) including executive processes such as the manipulation of attention or the shifting from interoceptive to exteroceptive attention (for example, within dual tasks or between tasks) [2,3]. IA can be modulated and can be trained by mindfulness training (MT): specifically, it seems that MT increases the contribution of interoception to perceptual experience [6].

In previous theoretical accounts, the interoceptive ability has been linked to the observation of pain experienced by another individual and to both cognitive and affective empathy components [8]. Affective empathy refers to any emotion that focuses on the other person's well-being, and hence to empathic concern, which is related to compassion. Cognitive empathy refers to the capacity to comprehend others' feelings or, more broadly, other people's perspectives [9]. It has been shown that, when faced with the observation of painful visual stimuli, a greater ability to perceive one's internal body states was linked to a higher estimated pain intensity (evidence con- 
sidered as a marker of cognitive empathic response), as well as a higher level of arousal and compassion feelings (finding interpreted as a marker of affective empathic response) [8]. In fact, individuals recreate their body's representations of pain when they observe pain in others due to joint activation of the pain matrix and empathy neural pathways, which results in stronger empathic reactions $[10,11]$. In line with this assumption, Bowling and colleagues [12], claimed that directing one's attention to internal physiological changes might improve conscious vicarious pain perception (supported by overactivity in brain regions involved in mirroring the states of others, such as somatosensory cortex for observed touch) in adults, despite the fact that the authors did not examine this impact on a neurophysiological level. Therefore, it is possible that manipulating IA might magnify the normal mirroring effect, as well as both empathy for pain and the corresponding brain processes elicited by witnessing pain in others.

Neuroscientific literature on the emotional and sensory neural correlates of observing the painful state of others (i.e., observation of pain in others) has been differentiated from research that deepened the direct experience of physical pain (i.e., painful stimulation) [13-16]. The observation of others' pain activates mirror neural networks, which allow us to comprehend a painful situation by stimulating the same brain pathways as if we were personally experiencing the feelings and emotions related to pain [17]. This phenomenon has been noted by the perception-action model of empathy as an anticipated perception of the condition of the subject receiving painful stimulation, which automatically triggers the representation of the observer on the condition of the observed subject: this representation automatically induces the corresponding autonomous and somatic reactions in the observer [18]. Nevertheless, the observation of pain in someone else does not automatically trigger the somatic correlates of pain because it is primarily a mental experience, in which empathy plays a key role in inducing emotional and cognitive representations of the situation $[16,19]$.

Neuroimaging research also analyzed the brain processes and cortical basis beneath the observation of pain in others [13-15]. A comprehensive body of work has shown the role of primary and secondary somatosensory cortices and motor regions in underlying the sensory qualities of others' pain [20]. Also, the somatosensory cortex shows a hemispheric specialization: the left side activation correlates to pain sensation [21] and empathy in situations involving nonphysical contact [22], while the right side is related to empathy in circumstances linked to physical touch [22]. More specifically, empathizing with others' pain appears to activate the whole pain matrix, including a group of frontal areas that are particularly engaged in the affective and motivational pain assessment, as well as the cognitive attentional dimension of pain [23-25]. Brain evidence indicates that empathy for pain observed in others triggers brain portions that are not commonly associated with pain perception, but mainly to processes of social cognition, such as interoception, emotional regulation, and social-cue processing [17].

Among other brain regions, it has been found that the dorsolateral prefrontal cortex (DLPFC) is activated by observing pain in others and is specialized in emotional regulation towards salient emotional stimuli [26]. It was also related to strong subjective empathy and, therefore, might indicate protective mechanism against emotional hyper-arousal [27]. In terms of hemispheric lateralization, earlier research has linked both left and right DLPFC activation to the downregulation of self-pain perception [28-30] and this modulation appears to be linked to its primary function on general emotional regulation processes, such as cognitive reappraisal and attention modulation of the affective response [31]. Different emotional regulation methods are related with lateralized DLPFC activity, according to Ochsner, Silver, and Buhle [31]: whereas the left side is commonly engaged in meaning reinterpretation of the affective response, the right side appears to play a role in psychological distancing from the emotional input.

Previous studies have mostly used neuroimaging techniques, such as functional Magnetic Resonance Imaging (fMRI) and magnetoencephalography to explore empathy for pain phenomenon $[15,32]$. Compared to those techniques, functional Near-Infrared Spectroscopy (fNIRS) is a relatively recent, non-invasive, and less expensive neuroimaging method capable of measuring cortical activity in a natural position [33]. It measures variations in the concentration of oxygenated and deoxygenated hemoglobin $(\mathrm{O} 2 \mathrm{Hb}$ and $\mathrm{HHb}$, respectively) to track regional cerebral blood flow (rCBF) [34]. Because of its high temporal resolution, its greater spatial resolution with respect to EEG, and the sensitivity to hemodynamic changes, fNIRS appears to be an excellent tool for studying the dynamically changing representation and integration of the empathic response among complex, extended brain networks [35]. In healthy people, fNIRS has been shown to be effective at distinguishing cortical responses to painful stimuli [36].

Based on previous evidence, two questions arise spontaneously. Could DLPFC and somatosensory activations related to the observation of pain in others be modulated by the conscious focus on one's interoceptive correlates? How can different types of stimuli (pain, body part, contextual cues) influence those brain activations? While, on the one hand, the face is a highly salient stimulus in human interactions, on the other hand, hand stimuli have been widely exploited for evoking the physical sensation of pain. Also, the social complexity of the background in which a pain stimulus is presented could influence its perception, and perceived agency was shown to affect empathy for pain [37]. In studies focusing on observation of pain in others', the stimuli are often presented in the individual condition (e.g., a single part of the body that receives painful stimulation) or in a social condition (e.g., two persons in an interactive dynamic, a person who causes physical pain to another person), and this factor is not usually controlled. Yet, it could be an intervening aspect 
because of the increase of complexity of the scene. A previous study highlighted that the observation of pain in socially contextualized scenes led to a specific hemodynamic increase in frontal and temporoparietal cortical regions involved in representing social interaction, emotion regulation, and perceived agency. However, in the set of stimuli adopted in that study the pain stimulation could be self-inflicted or inflicted by the other and it did not consist solely in perceiving the presence of another individual [37]. Limited previous studies explored empathy by using stimuli including the social context or consisting of interpersonal situations, a gap in the literature that the present study intends to fill.

Given these premises, this study aims to explore if and how the manipulation of IA modulates the neural correlates of emotional and sensory processes occurring during observation of pain in others. The effects linked to emotional, and sensory representations of observation of pain in others are supposed to be amplified by the manipulation of IA in the experimental group, which was explicitly asked to pay attention to interoceptive feelings while observing a set of painful/non-painful stimuli, confronted to the control group, which was instructed to merely observe the stimuli.

Firstly, we hypothesized a higher DLPFC activation for the experimental group compared to the control group, since we expect that the increased attention towards the correlates of the affective empathic response in the experimental group could engage DLPFC for emotion regulation strategies. Secondly, we also expect higher brain responsiveness to face compared to hand stimuli, given the affective salience of faces. Thirdly, we suppose higher brain responsiveness to pain compared to non-painful stimuli. Fourthly, we expect these effects to be enhanced in the individual compared to the social condition, considering the mirroring effect of pain observation. To summarize, we predicted that IA would enhance the mirroring effect of pain observation, resulting in a distinct pattern of neural activity in which the most salient conditions, such as face and painful stimuli, compared to hand and non-painful stimuli, displayed in an individual condition could elicit stronger neural reactivity.

\section{Materials and methods}

\subsection{Sample}

Twenty participants $(1$ male, Mage $=23$; SDage $=2.93$ ) were involved in this study: all caucasian right-handed university students with a normal-to-corrected vision. Exclusion criteria encompass any physiological and pathological condition of acute or chronic pain, chronic illnesses, histories of seizures, pregnancy, any psychiatric or neurologic disorder, and cognitive deficits. Participants were randomly allocated to one of two groups: experimental (EXP) or control $(\mathrm{CNT})$. Groups were matched for age $\left(\mathrm{M}_{E X P}=23.27\right.$; $\left.\mathrm{SD}_{E X P}=3.64 ; \mathrm{M}_{C N T}=22.66 ; \mathrm{SD}_{C N T}=1.63\right)$ and individual differences in IS. Before the screening phase, all individuals provided their voluntary consent to take part in the study and signed a written informed consent form. No com- pensation was provided for their participation in the study. The study was approved by the local ethics committee of the Department of Psychology of the Catholic University of the Sacred Heart of Milan, Italy, and was carried out following the latest version of the Declaration of Helsinki.

\subsection{Heartbeat detection task}

For checking and controlling individual differences in IS, each subject completed the Heartbeat Detection task, which assessed participants' capacity to perceive heartbeats [4]. Participants were placed in a sound-proofed room and told to begin silently counting at the appearance of a starting cue on a computer screen (grey desktop) until a visual stop signal appeared. They counted their heartbeat for four distinct time intervals ( 25 seconds, 35 seconds, 45 seconds, 100 seconds), which were randomly presented to them. At the conclusion of each period, participants must enter the number of heartbeats counted. They were not permitted to monitor their pulse throughout the task, and they received no feedback on the length of the counting phases or the quality of their performance. By utilizing E-Prime 2.0 program (Psychology Software Tools Inc., Sharpsburg, PA, USA), the trials were showed randomly. Moreover, for the four intervals, their Heart Rate (HR) was monitored through a physiological recording tool (Biofeedback 2000-xpert, version 7.01, Schufried GmbH, Mödling, Austria) sampling at $500 \mathrm{~Hz}$. The system, via a photopletismographic sensor (4 $\mathrm{mm}$ diameter $\mathrm{Ag} / \mathrm{AgCl}$ electrode) placed in correspondence to the distal phalanx of the forefinger of the non-dominant hand was able to measure HR.

The Interoceptive Sensitivity Index (ISI) is a measurement of IS that was obtained from the HBD task by computing the mean of the 4 heartbeat perception intervals using Schandry's formula [4]:

$1 / 4 \quad \Sigma(1-(\mid$ recorded $\quad$ heartbeats-counted heartbeats $\mid) /$ recorded heartbeats).

This index ranges from 0 to 1 with higher values suggesting small deviations between counted and recorded heartbeats. An independent-samples $t$-test (IBM SPSS 25, IBM Corp., Chicago, IL, USA) was applied to control ISI homogeneity between groups and there were no significant variations in the ISI score $\left(\mathrm{M}_{E X P}=0.51 ; \mathrm{SD}_{E X P}=0.21\right.$; $\left.\mathrm{M}_{C N T}=0.62 ; \mathrm{SD}_{C N T}=0.15\right)($ all $p>0.05)$.

\subsection{Procedure}

Participants were situated facing a computer monitor positioned $70 \mathrm{~cm}$ from the subject for the experimental phase, which was conducted in a quiet room. E-Prime 2.0 program (Psychology Software Tools Inc., Sharpsburg, PA, USA) was used to display the stimuli on a laptop with a 15 -inch screen, with a visual horizontal angle of $4^{\circ}$ and a vertical angle of $6^{\circ}$. To present the experimental procedure, each participant was given a systematic set of instructions.

During the recording of fNIRS hemodynamic data, participants were required to observe a set of stimuli. The sample, which had previously been divided into the EXP and CNT 
groups, was instructed to observe each stimulus and then rate them, on the computer keyboard, by selecting the letter " $\mathrm{K}$ " for pain stimuli and " $\mathrm{L}$ " for no pain stimuli on the computer keyboard (with the right index or middle finger), to provide the behavioral response. To manipulate IA, the EXP group was asked to concentrate on their interoceptive variations during stimuli observation, and they were given the following instructions: "During this task, we ask you to focus your attention on your bodily sensations. Try to observe how you feel and if there are any variations in your body as you look at the pictures". Orally we added a further suggestion about focusing for instance on the breath, as a further specification of written delivery. The CNT group was not specifically instructed to focus its attention on the interoceptive correlates, while receiving generic instructions to observe the stimuli and assess them for pain and no-pain.

The images were shown randomly for 5 seconds in the center of the screen, with a 10-second inter-stimulus delay during which participants fixed their gaze on a central cross. To avoid any bias related to sequence effects, the order of stimulus presentation was counterbalanced. The 160 stimuli were separated into four blocks, each with 40 stimuli. During the period after the stimulus had disappeared from the monitor, participants may submit behavioral responses for painful and non-painful stimulus features (for a graphical representation of the procedural steps, see Fig. 1, Ref. [38]). Final manipulation checks were used at the end of the task, and participants reported to be completely conscious of the task and indicated the amount of attention they gave to their physiological feelings throughout this debriefing. They were asked to tell how much they feel they have perceived changes in their body (such as the breath) during the task and the experimental group self-reported higher perception of this bodyrelated signal.

\subsection{Stimuli}

The stimuli included 32 images of a person (male or female, randomized) experiencing painful (needle penetration) or non-painful (Q-tip touch) stimulation in two separate body parts (face or hand). Pictures were also representing a single person (individual condition; a single person that receives a painful stimulation in a specific part of the body) either two persons in social interaction (social condition; two persons in an interactive dynamic, a person who causes physical pain to another person) both for the pain and body part set of stimuli (Fig. 2A-D). The two individuals that are part of the social scene could clearly be distinguished from one another. Each image lasted 5 seconds and portrayed persons with neutral facial expressions at a visual angle of $21^{\circ}$ $\times 17^{\circ}$ (width $\times$ height) at a viewing distance of $80 \mathrm{~cm}$. The following perceptual qualities of the stimuli were examined: position, size, brightness, content, content complexity, and comprehensibility. Using an easy modified 5-point version of the Self-Assessment Manikin scale (SAM) [39], a group of independent judges, controlled for gender and age, evaluated the emotional neutrality of the stimuli before needle penetration/Q-tip contact (i.e., pain/no-pain) attribution. Stimuli were assessed with average values for emotional valence $(\mathrm{M}=2.52 ; \mathrm{SD}=0.58)$ and arousal $(\mathrm{M}=2.72, \mathrm{SD}=$ 0.43). Emotional involvement towards the stimuli was controlled through a manipulation check. After the experimental task, participants were asked explicitly to tell if they felt emotionally involved with each category of stimuli (including individual/social distinction) ("Have you felt any emotions looking at these images?”), and no differences were found.

\subsection{Acquisition and analysis of $f$ NIRS data}

The hemodynamic responses consisting of a change in $\mathrm{O} 2 \mathrm{Hb}$ and $\mathrm{HHb}$ concentrations were recorded using a NIRScout System (NIRx Medical Technologies, LLC, Los Angeles, California) composed of a 14-channel optodes matrix. By employing a fNIRS Cap, eight light sources/emitters and eight detectors were mounted over the scalp in accordance with the international 10/5 standard [40].

Emitters positions were: AF3-AF4, F5-F6, FCC3hFCC4h, and CCP5h-CCP6h. Detectors were positioned in AFF1h-AFF2h, F3-F4, FCC5h-FCC6h, and CCP3h-CCP4h. For contiguous optodes, the emitter-detector distance was preserved at $30 \mathrm{~mm}$ and near-infrared light with two wavelengths (760 and $850 \mathrm{~nm}$ ) was employed. The following channels were obtained in this manner: Ch1 (AF3-F3), Ch2 (AF3-AFF1h), Ch3 (F5-F3), Ch4 (AF4-F4), Ch5 (AF4$\mathrm{AFF} 2 \mathrm{~h})$, Ch6 (F6-F4) were consistent of the left and right DLPFC (Brodmann Area 9, BA9); Ch7 (CCP5h-FCC5h), Ch8 (CCP5h-CCP3h), Ch9 (CCP6h-FCC6h), Ch10 (CCP6hCCP4h), Ch11 (FCC3h-FCC5h), Ch12 (FCC3h-CCP3h), Ch13 (FCC4h-FCC6h), Ch14 (FCC4h-CCP4h) corresponding to left and right somatosensory areas (Brodmann Area 1, BA2, BA3) (see Fig. 3). In order to correlate our locations with Broadmann Areas, we analyzed the positions of the sources and detectors, as well as the region between them, that included the channel. Then we sought for the most appropriate underlying functional region and Brodmann Area. To accomplish this, we drew on a variety of sources, including references and online atlases (see for example [41-43]).

Variations in the concentrations of oxygenated $(\mathrm{O} 2 \mathrm{Hb})$ and deoxygenated $(\mathrm{HHb})$ hemoglobin were recorded continuously during the task using NIRStar Acquisition Software, starting with a 120 -second resting baseline. Signals obtained from the 14 NIRS channels were collected at $6.25 \mathrm{~Hz}$ sampling rate and processed and transformed with nirsLAB software (v2014.05; NIRx Medical Technologies LLC, 15Cherry Lane, Glen Head, NY, USA), according to their wavelength and position, yielding results for variations in $\mathrm{O} 2 \mathrm{Hb}$ and $\mathrm{HHb}$ concentrations for each channel, scaled in $\mathrm{mmol} \cdot \mathrm{mm}$. Each channel's raw $\mathrm{O} 2 \mathrm{Hb}$ and $\mathrm{HHb}$ data was digitally band-pass filtered at $0.01-0.09 \mathrm{~Hz}$ [44]. Raw time series were visually screened for noisy channels (e.g., high motion errors or abrupt amplitude fluctuations), removing channels with poor optical coupling, such as the absence of $1 \mathrm{~Hz}$ heartbeat oscillations in raw data [45]. The mean concentration of each channel was established by averaging data across trials, and an aver- 


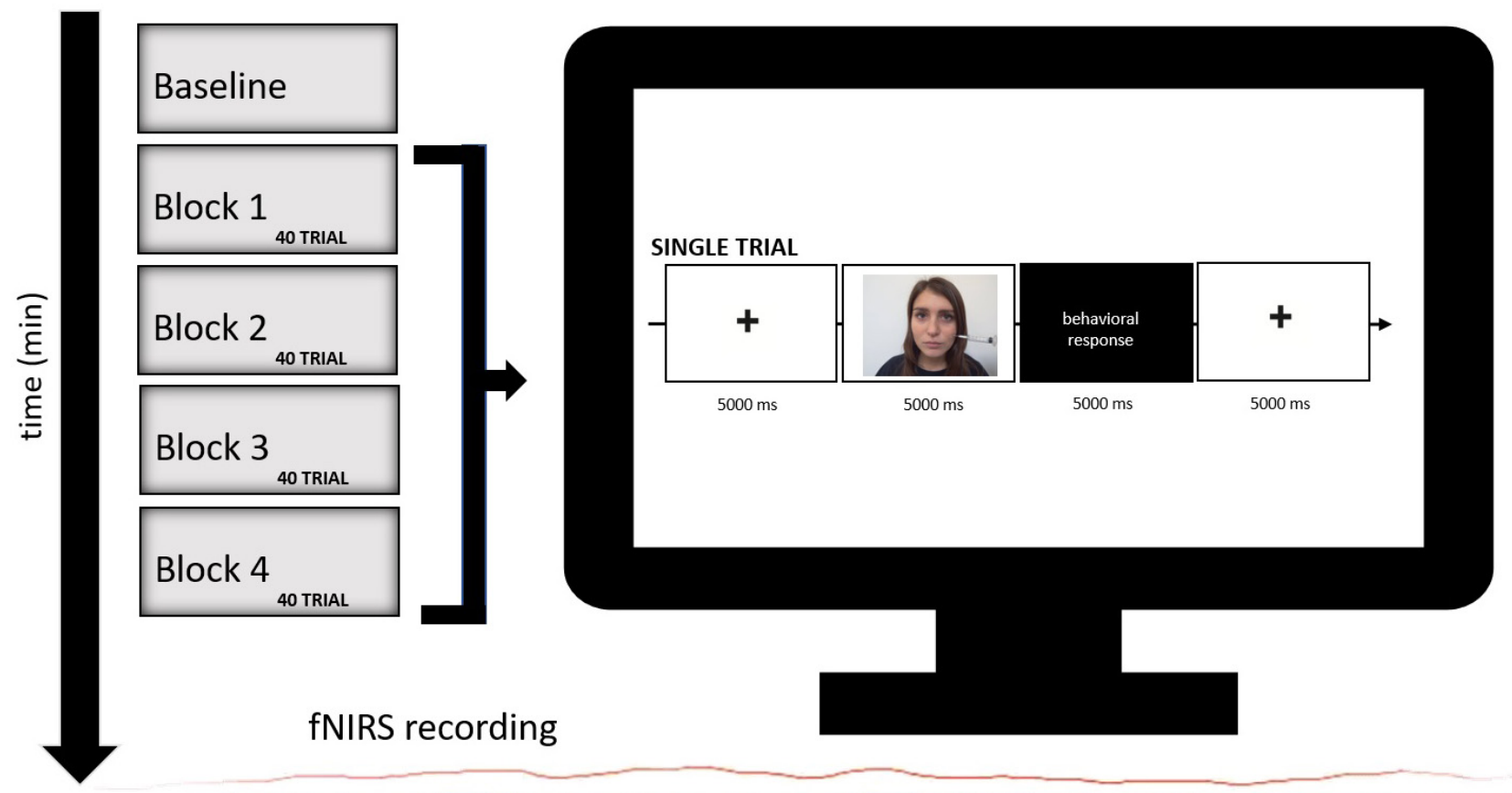

Fig. 1. Set up of the experiment. The figure describes fNIRS registration when participants were performing the observation of pain in others' task in the experimental setting and the single trial procedure (retrieved and modified from [38]).
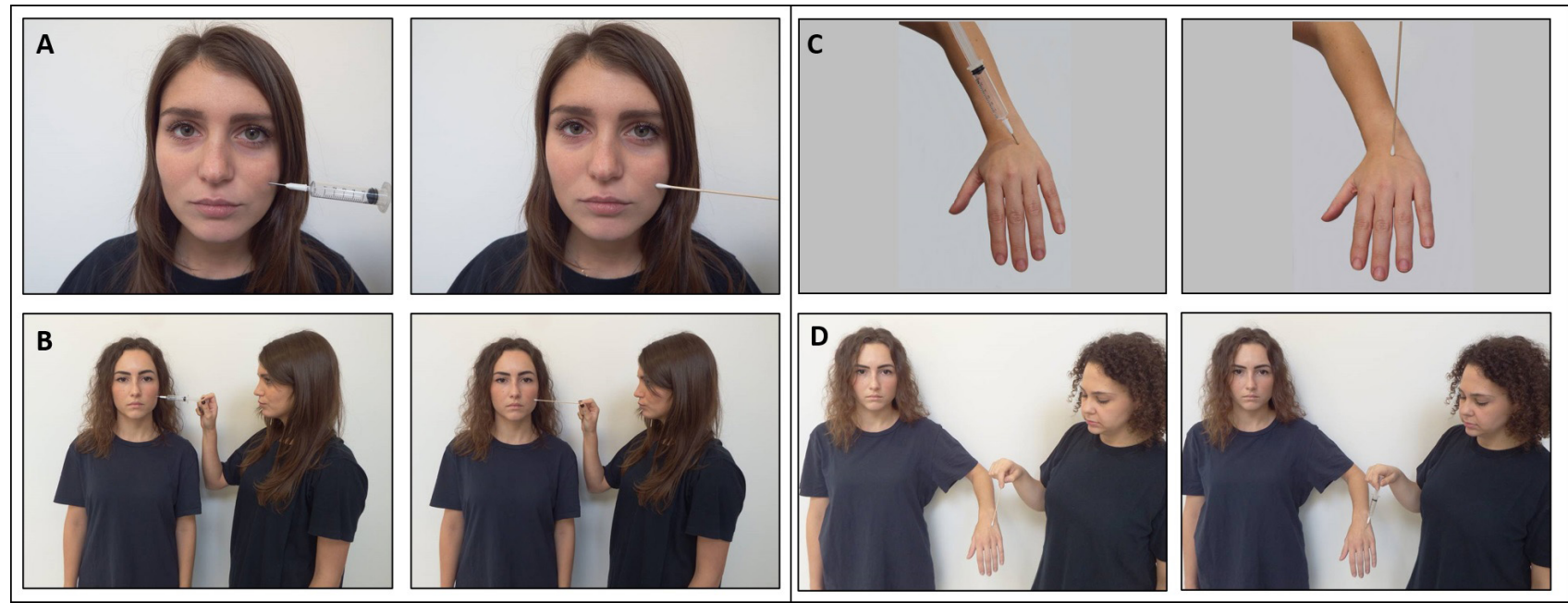

Fig. 2. Set of stimuli adopted in the study. Stimuli samples for painful (left) and non-painful (right) stimulation of the face in the (A) individual condition (B) social condition; and for painful (left) and non-painful (right) stimulation of the hand in the (C) individual condition (D) social condition.

age value for each condition was determined for the next 6 seconds starting with the stimulus onset. The effect size in each condition was determined based on the mean concentrations in the time series for each channel and subject. The effect sizes (Cohen's d) were computed by dividing the difference between the averages of the baseline and trial by the baseline standard deviation $(\mathrm{sd}): \mathrm{D}=(\mathrm{m} 1-\mathrm{m} 2) / \mathrm{s}$, where $\mathrm{m} 1$ and $\mathrm{m} 2$ are the mean concentration levels for baseline and trial, respectively, and $\mathrm{s}$ is the SD of the baseline. To improve the signal-to-noise ratio, the effect sizes from the 14 channels were averaged. Despite the fact that fNIRS raw data were relative values that could not be directly averaged across participants or channels, effect sizes normalized data could be averaged regardless of the unit since the effect size is unaffected by differential pathlength factor (DPF).

Factors such as the two Regions of Interest (ROI) combining the Frontal (F), and Central (C), left/right corresponding channels, as well as lateralization (left/right hemisphere), were included in the statistical analysis of the data. The values obtained from Ch1-Ch2-Ch3 and Ch4-Ch5-Ch6 were 


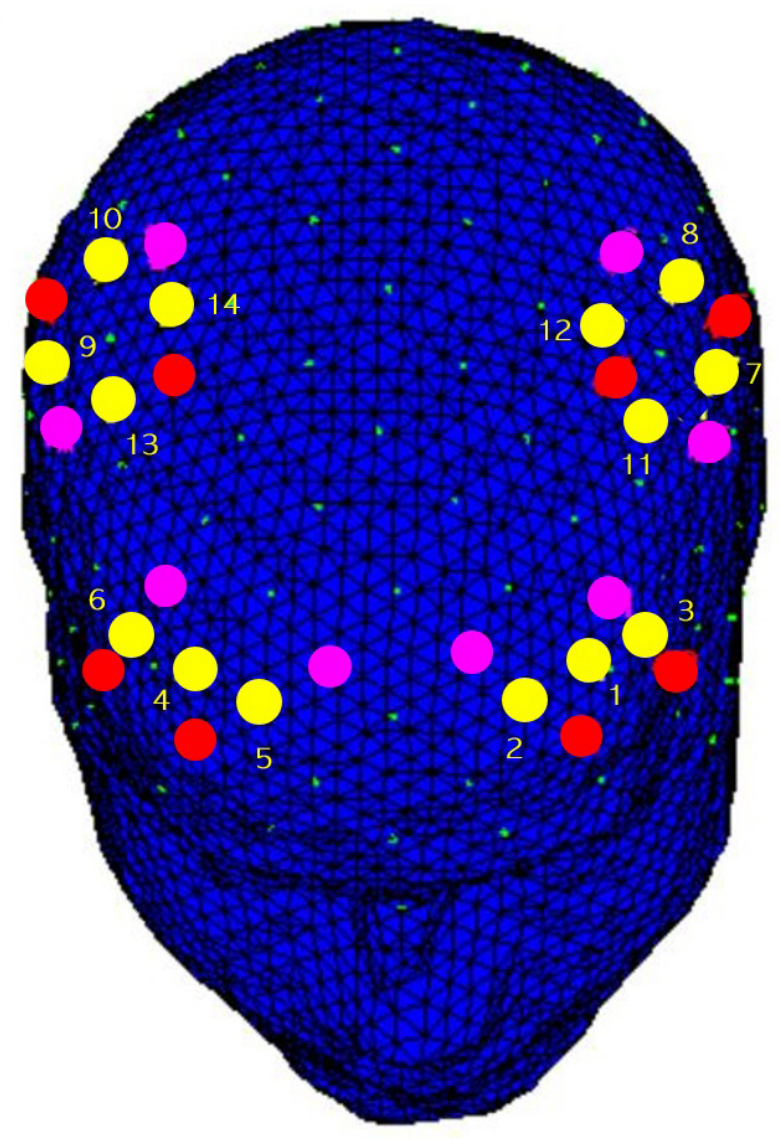

Fig. 3. fNIRS montage. The locations of the fNIRS montage including sources (red) and detectors (violet). Sources were in the subsequent positions: AF3-AF4, F5-F6, FCC3h-FCC4h, and CCP5h-CCP6h. Detectors were located on: AFF1h-AFF2h, F3-F4, FCC5h-FCC6h, and CCP3hCCP4h. The following 14 channels (yellow) were obtained: Ch1 (AF3F3), Ch2 (AF3-AFF1h), Ch3 (F5-F3), Ch4 (AF4-F4), Ch5 (AF4-AFF2h), Ch6 (F6-F4), Ch7 (CCP5h-FCC5h), Ch8 (CCP5h-CCP3h), Ch9 (CCP6hFCC6h), Ch10 (CCP6h-CCP4h), Ch11 (FCC3h-FCC5h), Ch12 (FCC3hCCP3h), Ch13 (FCC4h-FCC6h), Ch14 (FCC4h-CCP4h).

averaged for the activity of the left and right DLPFC regions, respectively, for the Frontal ROI. While for the Central ROI, the values acquired from Ch7-Ch8-Ch11-Ch12 and Ch9-Ch10-Ch13-Ch14 were averaged to reflect of the activity of the left/right somatosensory regions, respectively.

\subsection{Statistical data analysis}

Behavioral data consisting of response accuracies (ACC) were collected using E-prime Software for each subject during the task. ACC was calculated as the percentage of correct responses on the total responses for pain/non-pain stimuli. Then, to examine groups differences, an independentsamples $t$-test (IBM SPSS 25, IBM Corp., Chicago, IL, USA) was used. The statistical significance level was set to $\alpha=0.05$. Levene's test was used to check the equality of variances between groups and to adjust the calculation of subsequent inferential tests accordingly.
Regarding fNIRS data, two mixed repeated measures ANOVAs with independent within factors Pain (2: Pain, No Pain) $\times$ Condition (2: Individual, Social stimuli) $\times$ Body part $(2$ : Face, Hand $) \times$ Lateralization $(2$ : Left, Right $) \times$ Region of Interest (ROI) (2: Frontal, Central), and as between factor, the Group (2: EXP vs CNT) was applied to dependent fNIRS data $(\mathrm{O} 2 \mathrm{Hb}$ and $\mathrm{HHb}$ concentration values). The degrees of freedom have been adjusted using Greenhouse-Geisser epsilon where suitable for all ANOVA tests. The statistical significance level was set at $\alpha=0.05$. In the case of significant effects, pairwise comparisons were performed on the data. Pair-wise comparisons were performed to double-check simple effects for significant interactions, and Bonferroni correction was employed to eliminate multiple comparisons possible biases. In addition, the normality of the data distribution was checked using the kurtosis and asymmetry indices. Partial eta squared $\left(\eta^{2}\right)$ indices was used to evaluate the magnitude of statistically significant effects.

\section{Results \\ 3.1 Behavioral accuracy data}

Behavioral ACC was high for both groups. The accuracy for painful stimuli was 99\% for EXP and 96\% for the CNT group, whereas for non-painful stimuli, it was $99 \%$ for EXP and $100 \%$ for the CNT group. No significant differences were found for ACC (all $p>0.05$ ).

\section{2 fNIRS hemodynamic data}

The following results concern the statistical steps performed on $\mathrm{D}$ dependent measures for $\mathrm{O} 2 \mathrm{Hb}$ and $\mathrm{HHb}$ concentration values.

\subsection{1 $\mathrm{O} 2 \mathrm{Hb}$ values}

For the $\mathrm{O} 2 \mathrm{Hb}$ signal a first interaction effect Body part $\times$ ROI was found $\left(F_{[1,18]}=4.692, p \leq 0.044, \eta^{2}=0.207\right)$. Pairwise comparisons revealed significant higher mean values for face in frontal compared to central ROI $\left(F_{[1,18]}=7.075, p\right.$ $\left.\leq 0.016, \eta^{2}=0.282\right)$. Moreover, higher mean values were found for face compared to hand in frontal ROI $\left(F_{[1,18]}=\right.$ 7.148, $p \leq 0.015, \eta^{2}=0.284$ ).

A second interaction effect Condition $\times$ Body part $\times$ ROI was detected for $\mathrm{O} 2 \mathrm{Hb}\left(F_{[1,18]}=10.356, p \leq 0.005, \eta^{2}=\right.$ 0.365) (Fig. 4A-C). Pairwise comparisons showed a higher activation in the frontal compared to central ROI for face in the individual condition $\left(F_{[1,18]}=8.832, p \leq 0.008, \eta^{2}\right.$ $=0.329)$, and for face compared to hand in the frontal ROI in the individual condition $\left(F_{[1,18]}=0.066, p \leq 0.001, \eta^{2}=\right.$ 0.329 ). Moreover, significant greater activation was found in the frontal ROI for hand stimuli in the social compared to individual condition $\left(F_{[1,18]}=12.918, p \leq 0.002, \eta^{2}=0.418\right)$ No other significant effects were found.

\subsubsection{HHb values}

Regarding the $\mathrm{HHb}$ signal, four significant interaction effects were found and will be reported below. 
A
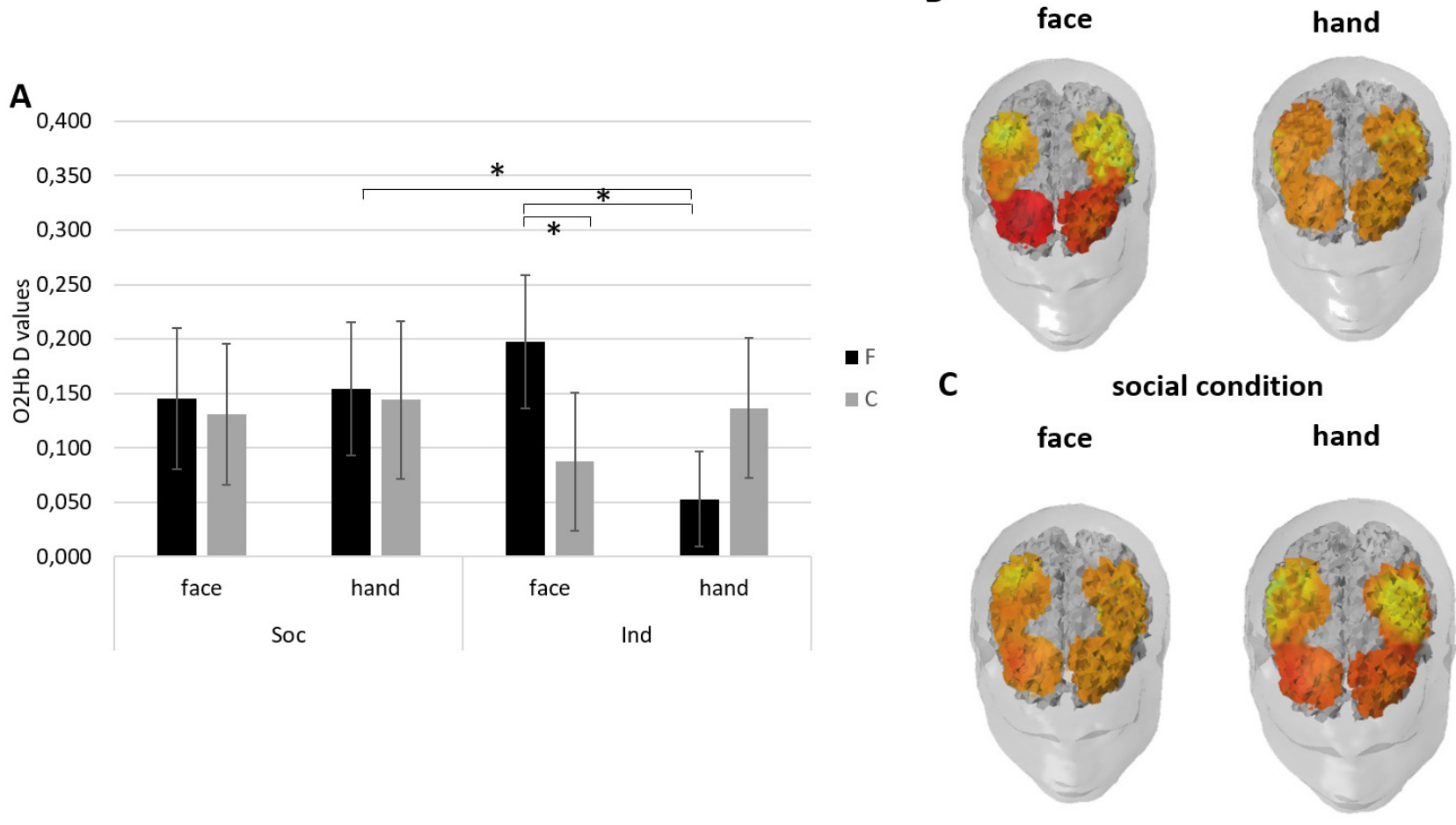

Fig. 4. Oxygenated hemoglobin results. (A) The bar charts show $\mathrm{O} 2 \mathrm{Hb} \mathrm{D}$ values for condition $\times$ body part $\times$ ROI interaction. Data are represented as mean \pm SE; all asterisks mark statistically significant differences, with $p \leq 0.05$. (B) Heads representation for O2Hb. The red area represents the increase of $\mathrm{O} 2 \mathrm{Hb}$ in the individual condition in the frontal areas for the face (left head) compared to hand (right head). (C) In the head displays, the red area represents the increase of $\mathrm{O} 2 \mathrm{Hb}$ in the social condition in the frontal areas for hand (right head) compared to face (left head).

Abbreviations. F, frontal; C, central; Ind, individual condition; Soc, Social condition.

Firstly, it was observed a significant interaction effect for Condition $\times$ Bodypart $\left(F_{[1,18]}=6.807, p \leq 0.018, \eta^{2}=0.274\right)$ (Fig. 5A). Specifically, pairwise comparisons showed a significant decrease in deoxy signal for face confronted with hand stimuli presented in the individual condition $\left(F_{[1,18]}=7.279\right.$, $p \leq 0.015, \eta^{2}=0.288$ ). In addition, according to pairwise comparisons, this $\mathrm{HHb}$ decrease for face stimuli is significantly more enhanced in the individual than social condition $\left(F_{[1,18]}=7.389, p \leq 0.014, \eta^{2}=0.291\right)$ (Fig. 4B-C).

A second interaction effect emerged regarding Condition $\times$ Pain $\left(F_{[1,18]}=5.050, p \leq 0.037, \eta^{2}=0.219\right)$. A significant decrease in deoxy signal was identified in the individual condition for pain compared to no pain stimuli $\left(F_{[1,18]}=6.562, p\right.$ $\leq 0.020, \eta^{2}=0.267$ ) (Fig. 5B-C). Also, pairwise comparisons showed lower $\mathrm{HHb}$ values for painful stimuli in the individual compared to social condition $\left(F_{[1,18]}=5.063, p \leq 0.037\right.$, $\eta^{2}=0.220$ ).

Thirdly, an interaction effect Bodypart $\times$ Group was detected $\left(F_{[1,18]}=6.042, p \leq 0.024, \eta^{2}=0.251\right)$. For the EXP group, a significant decrease of $\mathrm{HHb}$ values were found for the face compared to hand stimuli $\left(F_{[1,18]}=7.426, p \leq 0.014\right.$, $\left.\eta^{2}=0.292\right)$ as revealed by pairwise comparisons (Fig. 6A-B).

As fourth result, an interaction effect Condition $\times$ Lateralization was detected $\left(F_{[1,18]}=6.590, p \leq 0.019, \eta^{2}=0.268\right)$. Pairwise comparisons revealed a significant decrease of $\mathrm{HHb}$ signal in the right compared to left hemisphere in the social condition $\left(F_{[1,18]}=6.051, p \leq 0.024, \eta^{2}=0.252\right)$. Moreover, significant lower $\mathrm{HHb}$ values were found in the left hemisphere in the individual versus social condition $\left(F_{[1,18]}\right.$ $=4.789, p \leq 0.042, \eta^{2}=0.210$ ) (Fig. 6C-D).

\section{Discussion}

This research offered novel evidence on the cortical activation and hemodynamic response of DLPFC and somatosensory areas in the context of empathy for pain thanks to the application of fNIRS. Specifically, in this context, IA was modulated to observe the effects of the focus on body correlates on targeted stimuli perception. The sample was divided into an EXP group, asked to pay attention to the interoceptive correlates, and a CNT group not receiving direct interoception instructions. During the experiment, participants were asked to observe painful/non-painful stimuli belonging to different body parts (face vs hand), displayed in individual or social conditions.

The main findings will be discussed below. Firstly, both groups showed higher DLPFC activation for face stimuli presented in the individual condition confronted to hand stimuli in the social condition. Secondly, in the individual condition higher brain responsiveness was detected for pain confronted to non-painful stimuli. Thirdly, a relevant activation for face 

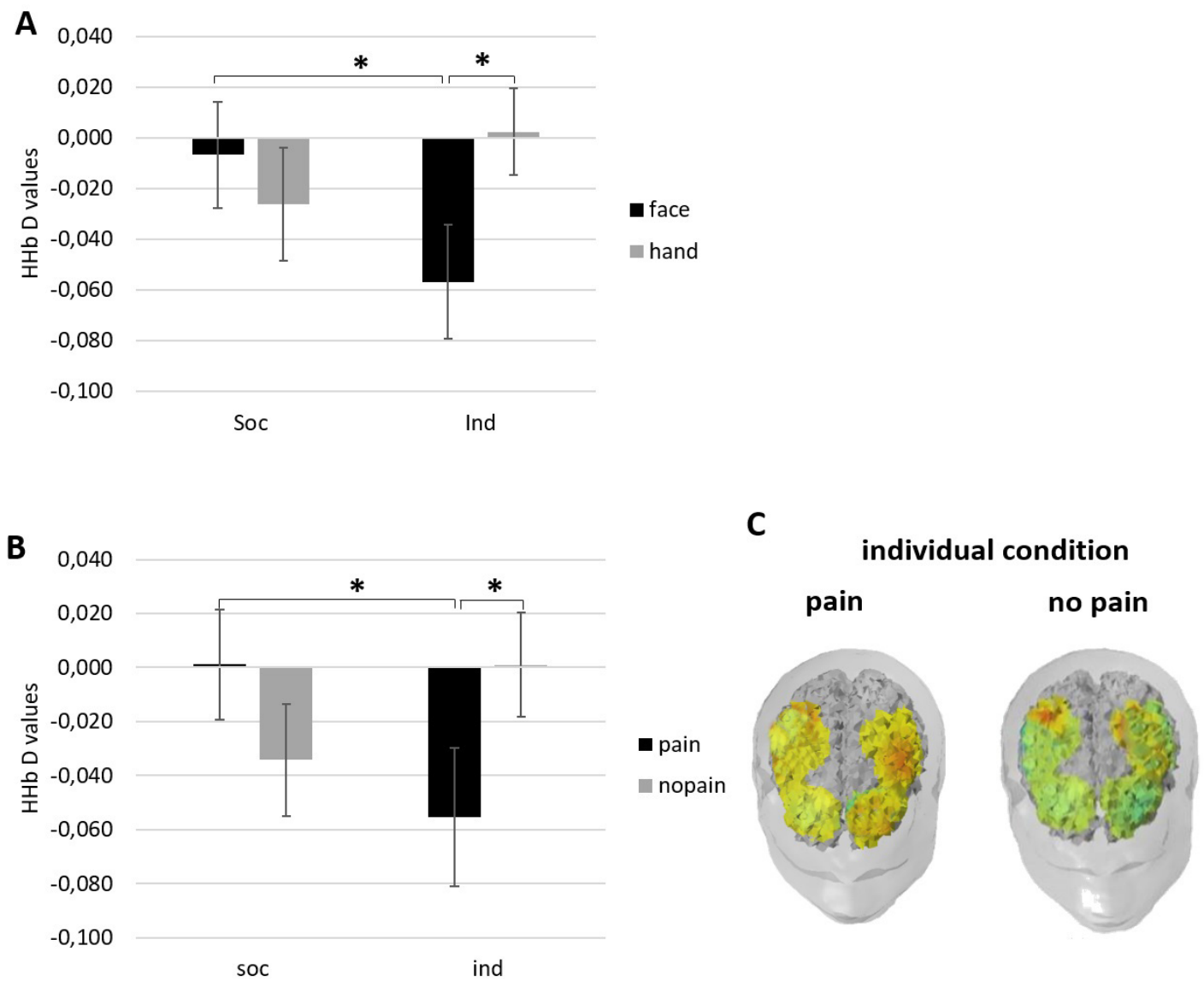

Fig. 5. Deoxygenated hemoglobin results. (A) The bar chart shows $\mathrm{HHb} \mathrm{D}$ values for condition $\times$ body part interaction. (B) Bar graph shows $\mathrm{HHb} \mathrm{D}$ values for condition $\times$ pain interaction. (C) The head displays show the significant activation (decrease of HHb) for pain (left head) compared to no-pain stimuli (right head) in the individual condition. For all charts, data are represented as mean \pm SE; all asterisks mark statistically significant differences, with $p$ $\leq 0.05$.

Abbreviations. Ind, individual condition; Soc, Social condition.

stimuli was mainly detected for the EXP group, suggesting that this phenomenon has certain features, namely, it manifests itself in the individual condition and for pain stimuli, it mostly activates all subjects, but above all the EXP group that is focusing on their interoceptive correlates. Lastly, a left/right hemispheric lateralization effect was found for the individual and the social condition, respectively.

Starting with the effects found for the oxygenated hemoglobin, firstly, an increase of $\mathrm{O} 2 \mathrm{Hb}$ was detected for both groups in the frontal areas for face stimuli. The face emerged as the most relevant body part, and it mainly activates anterior regions compared to central ones. Moreover, this frontal activation is mainly enhanced for face compared to hand stimuli and partially confirmed our second hypothesis. This frontal activation could be justified by the role that DLPFC plays in the modulation of emotional responses.

According to Ray and Zald [46], the DLPFC is thought to be involved in cognitive reappraisal, which is the adjustment of an emotional response based on one's cognitive reinterpretation of emotional information. Therefore, DLPFC may act as a regulatory structure of emotional response directed towards a highly significant stimulus [47-49]. In human relationships, the face is a significant social stimulus [5054]. Face processing may be separated into a first perceptive phase, in which the person completes the "structural codes" of face and a second phase in which the subject completes the "expression code" implicated in the decoding of emotional facial expressions [55]. The first is thought to be processed separately from complex facial information such as emotional meaning [56-61]. Here we argue that the simple presentation of a face receiving painful or non-painful stimulation in the context of pain observation in others activates frontal brain regions connected to emotional regulation of the empathic response, more than somatosensory areas.

Moreover, it is also noteworthy to observe that significant variations between ROI activation were found for face only and not for hand stimuli. A possible explanation for this absence of significant difference in ROI activation could be the strength of the social significance of face over the hand stimulus. Empathy for pain studies frequently adopted 
A
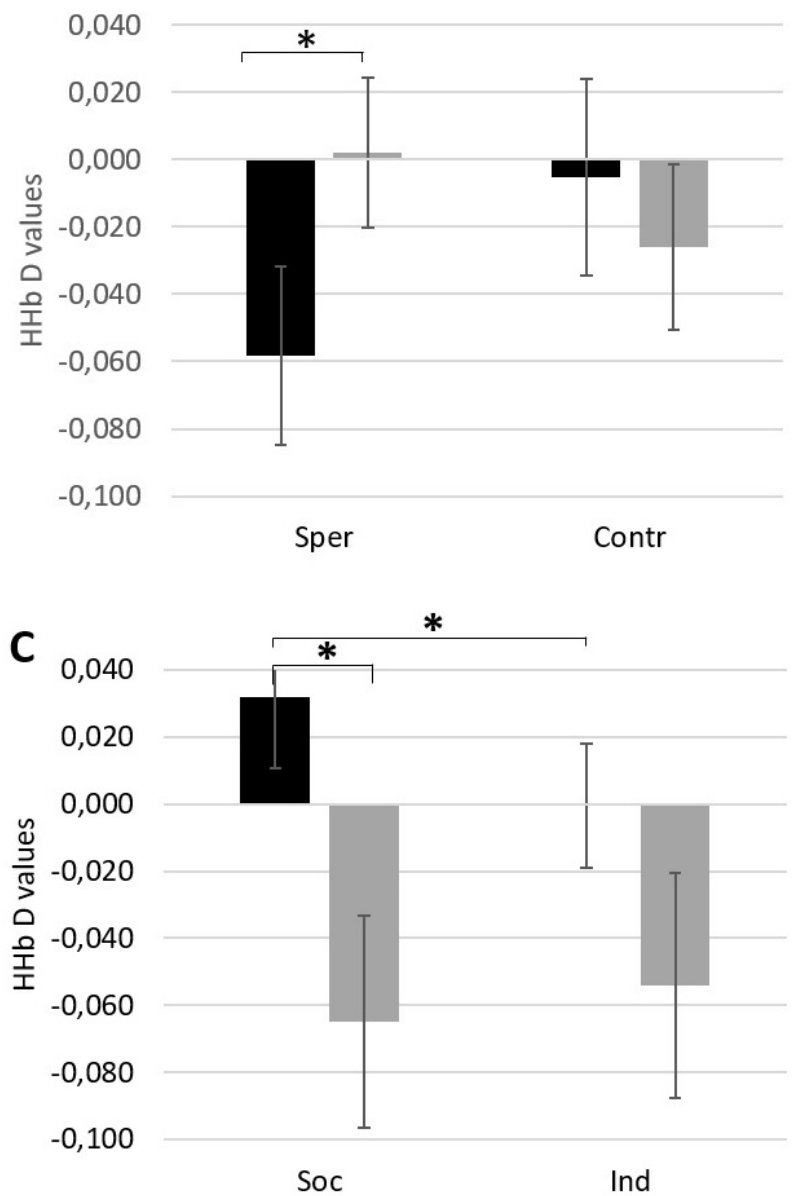

B
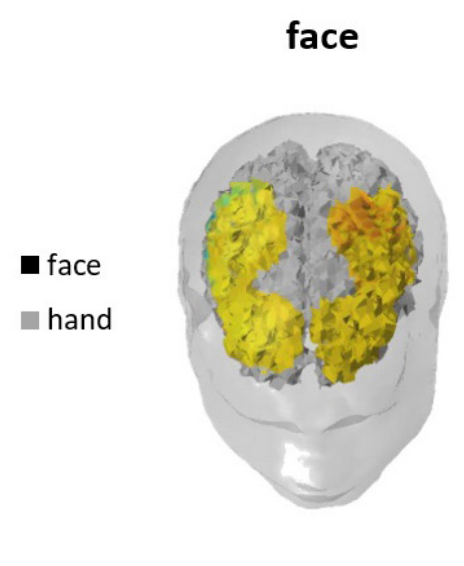

EXP group

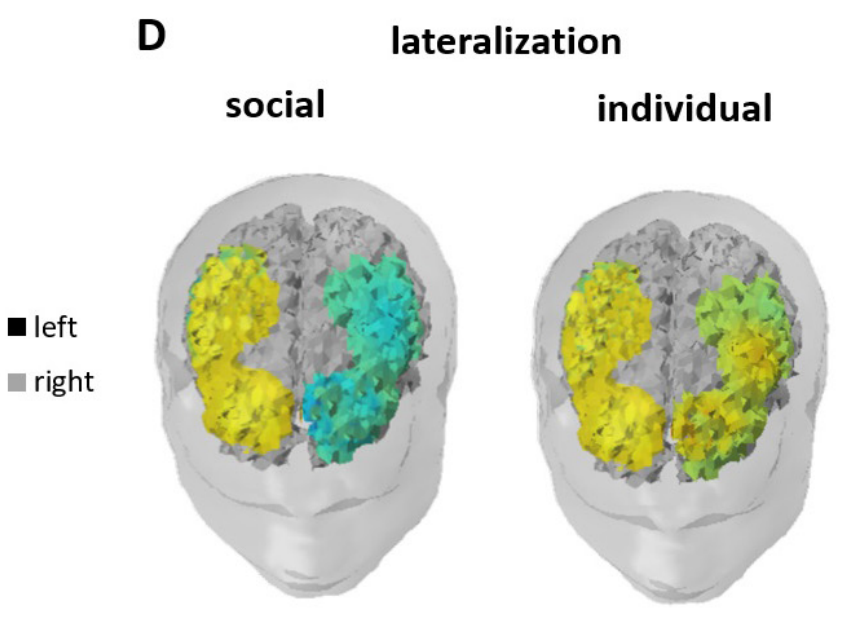

Fig. 6. Deoxygenated hemoglobin results. (A) The bar chart shows HHb D values for body part $\times$ group interaction. (B) Heads' representation of the increased activation in the EXP group for the face (left head) compared to hand stimuli (right head). (C) The bar graph shows HHb D values for condition $\times$ lateralization interaction. (D) In the head displays, it is represented the left hemisphere significant activation in the individual (right head) compared to the social condition (left head). For all charts, data are represented as mean \pm SE; all asterisks mark statistically significant differences, with $p \leq 0.05$.

Abbreviations. Sper, Experimental group; Contr, Control group; Ind, individual condition; Soc, Social condition.

body parts where pain stimulation was applied indistinctively $[13,26,62,63]$. However, here we argued that stimuli should be controlled and distinguished since face per se brings with it a "face effect" that specifically engages DLPFC.

This frontal activation for face stimuli, compared to central areas and compared to hand stimuli, has been significantly detected in the individual condition. This effect (face vs hand significance in the individual condition) was detected both for $\mathrm{O} 2 \mathrm{Hb}$ and $\mathrm{HHb}$ and could be considered as a specification of the previous one. Going down with the specifics, as previously mentioned, it appears in the individual condition that the frontal activation pattern for the face prevails, and prevails over the hand stimuli, thus suggesting the face has a strong widespread effect. Face it is a highly relevant stimulus, from early in life, people display a keen sensitivity to the occurrence of pain in others [64], and develop a highly refined ability to evaluate pain intensity from facial displays [65]. This ability usually develops in a one-to-one relation- ship between the newborn and the mother, which is a situation closer to the individual condition of face here depicted. Thus, when it comes to the process of recognizing pain in the face, it can be deemed that it is an individual process.

In the framework of observation of pain in others, the study of facial expression of pain has also found the involvement of brain areas subserving the direct experience of pain $[66,67]$. Present results have been examined in relation to "shared representations" theories of empathy, which claim that all empathic processes, from emotional contagion to cognitive empathy, start with a mapping of the expresser's perceived emotional state on a representation of the observer's equivalent condition [68]. When pain states are communicated via facial expression, the neurobiological substrate of this shared representation may encompass the human "mirror neuron" system [69], possibly via interactions with neural regions engaged in emotions [70]. However, empathy could potentially occur without the involvement of the classic mo- 
tor mirror mechanisms [71], but with the support of several brain areas that go beyond the motor system and classic pain matrix [72]. It follows that even face per se, given its highly informative nature concerning the status of the person, might have caught participants' attention, and activated frontal brain structures to modulate the empathic response.

Also, somatosensory resonance has been recently associated with empathy-related psychological closeness. In particular, the key activations seemed to be progressively decreased as a function of the psychological closeness between the participant and other models, with the strongest activation for the human models (which are the most psychologically close for the participants) [73]. Another possible interpretation could be that in the present study the DLPFC face-related activation in the individual condition was higher with respect to hand-related activation in social condition because the face-related individual (as it would be looking oneself in a mirror) was a psychologically closer condition (and closest with respect to all other conditions) compared to the psychologically farther condition (hand-related social), and therefore elicited stronger facilitation to incorporate the stimulus into the participants' body schema. A left DLPFC activation was formerly detected during the observation of pain in a close significant other [74]. It might be possible that the focused attention on one's interoceptive correlates might enhance the psychological closeness effect, however, further neuroimaging studies are needed to disentangle the link between interoception, empathy for pain and DLPFC activation.

Secondly, both groups were found to be more active (given a significant decrease in $\mathrm{HHb}$ signal) for pain compared to non-painful stimuli in the individual condition. This effect is in line with our third hypothesis and previous neuroimaging studies underlying that the observation of pain in others seems to recruit the whole pain matrix, including somatosensory cortices and a set of frontal regions particularly implicated in the affective and motivational evaluation of pain and the cognitive attentional dimension of pain [2325]. Moreover, this extensive activation for pain stimuli is significantly more enhanced in the individual condition compared to the social condition. Therefore, it is possible to state that the attentional focus is primarily directed towards painful face stimuli presented in the individual condition, as if the pain became more evident and effective in face stimuli presented alone: these (individual more than social and face more than hand) are the conditions in which pain in its salience emerges. Perhaps one possible explanation could be that pain is more effective when presented in an individual dimension, evidence that could support our fourth hypothesis.

Instead, regarding hand stimuli, there is a higher activation of frontal areas for hand presented in the social compared to the individual condition. It follows that in our study hand stimuli effect is evoked by the social context, that given its complexity, perhaps requires more cognitive effort, high- level executive function, and the recruitment of top-down cognitive control, as represented by DLPFC activation. It is possible to suggest that the social condition may function as a drive for the response in the frontal brain regions for hand stimuli, while the individual condition could evoke an increase of $\mathrm{O} 2 \mathrm{Hb}$ for face stimuli in the frontal areas. Therefore, on the one hand, the activation of the DLPFC for face stimuli in the individual condition suggests an effect of emotional regulation and predisposition of the empathic response in a typical relational situation, while, on the other hand, the DLPFC activation for the hand stimuli in the social condition suggests the effort to analyze a socially complex situation.

Thirdly, one of the most interesting effects that have been found was a decrease of $\mathrm{HHb}$ for the face compared to hand stimuli in the EXP group. This effect may suggest a relevant activation for face stimuli for the EXP group. Again, the keystone, in this case, is related to body-part, indeed the evidence that stands out above all is related to face. This "face effect" has certain features, namely, it manifests itself in the individual condition and for pain stimuli. It mostly activates all subjects and, above all, the EXP group, that is focusing on their interoceptive correlates.

A possible explanation of this result could be that in this group IA promoted the recruitment of internal adaptive regulatory strategies engaging both DLPFC and somatosensory areas towards a potentially emotionally relevant stimulus, thus partially confirming our first hypothesis, with a more widespread effect at the cortical level. To support this notion, in prior work Farb and colleagues [75] demonstrated that IA training reduces the involuntary and automatic cognitive elaboration, and promotes awareness of internal sensation and emotional experiences. In addition, this improvement in IA is associated with emotional regulation strategies, in order to control the emotional mood response in case of complex conditions [76].

Finally, a lateralization effect has been found for the $\mathrm{HHb}$ signal, for which the left hemisphere results more active for the individual condition compared to the social condition, 7 while the right side is more active compared to the left hemisphere in the social condition. Regarding the ROIs considered for this study, the left DLPFC activation was found to be involved with the meaning reinterpretation of the affective response [31] while the left somatosensory cortex correlates to pain sensation [21] and to empathy in situations related to nonphysical contact [22], and it was found to be active during empathy for pain task regardless of whether the participants observed painful or non-painful stimuli [77]. The right DLPFC activation was shown to play a role in psychological distancing from the emotional stimulus [31], while the right somatosensory cortex is associated with empathy in situations related to physical contact [22]. According to these previous studies, it might be plausible to state that the lefthemispheric activation in the individual condition is related to the cognitive regulation of the affective response towards stimuli where the physical contact is not strongly enhanced. 
For instance, facial pain stimuli in the individual condition require the adoption of regulatory strategies, thus engaging more the left part of the DLPFC, which supports the downregulation of empathic response to pain. Conversely, the right activation for the social condition could correspond to an analysis of the social context in which is evident the dual dimension and the physical contact between the two actors in the scenario.

As a methodological note, it is interesting to point out that in the present study most significant effects were also found for the deoxygenated hemoglobin. The HHb signal was here interpreted according to previous studies demonstrating that neural activation is commonly followed by an increase in fNIRS $\mathrm{O} 2 \mathrm{Hb}$ and a corresponding decrease in $\mathrm{HHb}$ signals [78]. Indeed, in studies comparing fNIRS to fMRI signal dynamic, it was shown how during stimulation, $\mathrm{O} 2 \mathrm{Hb}$ increases, whereas $\mathrm{HHb}$ decreases $[79,80]$, indicating an increase in $\mathrm{rCBF}$ and regional cerebral blood volume ( $\mathrm{rCBV}$ ) due to neurovascular coupling [81]. While, during the poststimulus period when rCBF returns to the baseline, $\mathrm{O} 2 \mathrm{Hb}$ was shown to decrease below, whereas $\mathrm{HHb}$ increases above baseline values [82]. Herein, a decrease of $\mathrm{HHb}$ signal has been interpreted as and results go in the direction of confirming previous $\mathrm{O} 2 \mathrm{Hb}$ effects, but also in providing additional interesting and relevant information.

This study is not without limitations. Indeed, firstly, independent verification of the effectiveness of interoceptive attentiveness manipulation should be included in future research, such as the inclusion of a Visual Analogue Scale (VAS) monitoring and checking the attention to the internal state at the end of each block. Secondly, future research should integrate a BIO-EEG-fNIRS recording to collect independent and implicit measures of emotional response to the different stimuli (in particular, about the individual/social condition, that is a peculiarity of this experimental design). Thirdly, due to the exploratory nature of this work, no sample size was estimated based on the statistical power. Consequently, the sample size must be expanded and matched for gender to validate and better generalize the observed findings. Because our sample was small and mostly composed of women, its generalizability and external validity are moderate and can be improved in forthcoming works. To the best of our knowledge, the literature on these processes is still limited and it would be interesting to check the effect of gender variable in further research.

\section{Conclusions}

To summarize, this study showed that, in the individual condition, higher brain responsiveness was detected for painful confronted to non-painful stimuli, and a left/right hemispheric lateralization was found for the individual and social condition, respectively. In addition, both groups showed higher DLPFC activation for face stimuli showed in the individual condition confronted with hand stimuli in the social condition. However, face stimuli activation prevailed for the EXP group, suggesting this phenomenon has certain features, namely it manifests itself in the individual condition and for pain stimuli. It is possible to conclude that IA promoted the recruitment of internal adaptive regulatory strategies engaging mainly DLPFC regions towards emotionally relevant stimuli.

\section{Abbreviations}

ACC, accuracies; ANOVA, Analysis Of Variance; C, Central; CNT, control group; DLPFC, dorsolateral prefrontal cortex; DPF, differential pathlength factor; EEG, electroencephalogram; EXP, experimental group; F, Frontal; fNIRS, functional Near-Infrared Spectroscopy; $\mathrm{HHb}$, deoxygenated hemoglobin; IA, interoceptive attentiveness; IS, Interoceptive Sensitivity; ISI, Interoceptive Sensitivity Index; M, Mean; MNS, mirror neuron system; $\mathrm{O} 2 \mathrm{Hb}$, oxygenated hemoglobin; rCBF, regional cerebral blood flow; ROI, Regions of Interest; SAM, Self-Assessment Manikin scale; SD, Standard Deviation.

\section{Author contributions}

MB and LA conceived and designed the experiments; MB and LA performed the experiment; MB and LA analyzed the data; MB and LA wrote the paper. All authors contributed to editorial changes in the manuscript. All authors read and approved the final manuscript.

\section{Ethics approval and consent to participate}

All participants provided their voluntary consent to take part in the study and signed the written informed consent form before the screening phase. All procedures performed in this study involving human subjects were following the ethical standards of the institutional research committee, Department of Psychology, Catholic University of the Sacred Heart, Milan, Italy, and with the 1964 Helsinki declaration and its later amendments or comparable ethical standards.

\section{Acknowledgment}

Not applicable.

\section{Funding}

This research received no external funding.

\section{Conflict of interest}

The authors declare no conflict of interest.

\section{References}

[1] Craig AD. How do you Feel? An interoceptive moment with your neurobiological self. New Jersey, USA: Princeton University Press. 2015.

[2] Critchley HD, Garfinkel SN. Interoception and emotion. Current Opinion in Psychology. 2017; 17: 7-14.

[3] Tsakiris M, De Preester H. The interoceptive mind: from homeostasis to awareness. Oxford, UK: Oxford University Press. 2018.

[4] Schandry R. Heartbeat detection and emotional experience. Psychophysiology. 1981; 18:483-488. 
[5] Ainley V, Brass M, Tsakiris M. Heartfelt imitation: high interoceptive awareness is linked to greater automatic imitation. Neuropsychologia. 2014; 60: 21-28.

[6] Farb NAS, Segal ZV, Anderson AK. Mindfulness meditation training alters cortical representations of interoceptive attention. Social Cognitive and Affective Neuroscience. 2013; 8: 15-26.

[7] Schulz SM. Neural correlates of heart-focused interoception: a functional magnetic resonance imaging meta-analysis. Philosophical Transactions of the Royal Society of London. Series B, Biological Sciences. 2017; 371: 20160018.

[8] Grynberg D, Pollatos O. Perceiving one's body shapes empathy. Physiology \& Behavior. 2015; 140: 54-60.

[9] Davis MH. Measuring individual differences in empathy: Evidence for a multidimensional approach. Journal of Personality and Social Psychology. 1983: 44, 113-126.

[10] Singer T, Lamm C. The social neuroscience of empathy. Annals of the New York Academy of Sciences. 2009; 1156: 81-96.

[11] Ernst J, Northoff G, Böker H, Seifritz E, Grimm S. Interoceptive awareness enhances neural activity during empathy. Human Brain Mapping. 2013; 34: 1615-1624.

[12] Bowling NC, Botan V, Santiesteban I, Ward J, Banissy MJ. Atypical bodily self-awareness in vicarious pain responders. Philosophical Transactions of the Royal Society B: Biological Sciences. 2019; 374: 20180361.

[13] Osborn J, Derbyshire SWG. Pain sensation evoked by observing injury in others. Pain. 2010; 148: 268-274.

[14] Vachon-Presseau E, Roy M, Martel MO, Albouy G, Chen J, Budell L, et al. Neural processing of sensory and emotionalcommunicative information associated with the perception of vicarious pain. NeuroImage. 2012; 63: 54-62.

[15] Lamm C, Decety J, Singer T. Meta-analytic evidence for common and distinct neural networks associated with directly experienced pain and empathy for pain. NeuroImage. 2011; 54: 2492-2502.

[16] Krishnan A, Woo C, Chang LJ, Ruzic L, Gu X, López-Solà M, et al. Somatic and vicarious pain are represented by dissociable multivariate brain patterns. ELife. 2017; 5: 1-42.

[17] Fitzgibbon BM, Giummarra MJ, Georgiou-Karistianis N, Enticott PG, Bradshaw JL. Shared pain: from empathy to synaesthesia. Neuroscience and Biobehavioral Reviews. 2010; 34: 500-512.

[18] Preston SD, de Waal FBM. Empathy: its ultimate and proximate bases. The Behavioral and Brain Sciences. 2002; 25: 1-71.

[19] Chang LJ, Gianaros PJ, Manuck SB, Krishnan A, Wager TD. A Sensitive and Specific Neural Signature for Picture-Induced Negative Affect. PLoS Biology. 2015; 13: e1002180.

[20] Betti V, Aglioti SM. Dynamic construction of the neural networks underpinning empathy for pain. Neuroscience and Biobehavioral Reviews. 2016; 63: 191-206.

[21] Bufalari I, Aprile T, Avenanti A, Di Russo F, Aglioti SM. Empathy for pain and touch in the human somatosensory cortex. Cerebral Cortex. 2007; 17: 2553-2561.

[22] Bolognini N, Rossetti A, Convento S, Vallar G. Understanding others' feelings: the role of the right primary somatosensory cortex in encoding the affective valence of others' touch. The Journal of Neuroscience. 2013; 33: 4201-4205.

[23] Apkarian AV, Bushnell MC, Treede R, Zubieta J. Human brain mechanisms of pain perception and regulation in health and disease. European Journal of Pain. 2005; 9: 463-484.

[24] Tracey I, Mantyh PW. The cerebral signature for pain perception and its modulation. Neuron. 2007; 55: 377-391.

[25] Hauck M, Lorenz J, Engel AK. Role of synchronized oscillatory brain activity for human pain perception. Reviews in The Neurosciences. 2008; 19: 441-450.

[26] Enzi B, Amirie S, Brüne M. Empathy for pain-related dorsolateral prefrontal activity is modulated by angry face perception. Experimental Brain Research. 2015; 234: 3335-3345.

[27] de Greck M, Shi Z, Wang G, Zuo X, Yang X, Wang X, et al. Culture modulates brain activity during empathy with anger. NeuroImage. 2012; 59: 2871-2882.

[28] Boggio PS, Zaghi S, Lopes M, Fregni F. Modulatory effects of an- odal transcranial direct current stimulation on perception and pain thresholds in healthy volunteers. European Journal of Neurology. 2008; 15: 1124-1130.

[29] Lorenz J, Minoshima S, Casey KL. Keeping pain out of mind: the role of the dorsolateral prefrontal cortex in pain modulation. Brain. 2003; 126: 1079-1091.

[30] Rêgo GG, Lapenta OM, Marques LM, Costa TL, Leite J, Carvalho $S$, et al. Hemispheric dorsolateral prefrontal cortex lateralization in the regulation of empathy for pain. Neuroscience Letters. 2015; 594: 12-16.

[31] Ochsner KN, Silvers JA, Buhle JT. Functional imaging studies of emotion regulation: a synthetic review and evolving model of the cognitive control of emotion. Annals of the New York Academy of Sciences. 2012; 1251: E1-E24.

[32] Levy J, Goldstein A, Pratt M, Feldman R. Maturation of Pain Empathy from Child to Adult Shifts from Single to Multiple Neural Rhythms to Support Interoceptive Representations. Scientific Reports. 2018; 8: 1810.

[33] Balconi M, Molteni E. Past and future of near-infrared spectroscopy in studies of emotion and social neuroscience. Journal of Cognitive Psychology. 2016; 28: 129-146.

[34] Pinti P, Tachtsidis I, Hamilton A, Hirsch J, Aichelburg C, Gilbert S, et al. The present and future use of functional near-infrared spectroscopy (fNIRS) for cognitive neuroscience. Annals of the New York Academy of Sciences. 2020; 1464: 5-29.

[35] Ogawa T, Nomur M. Using NIRS to investigate social relationship in empathic process. Infrared Spectroscopy - Life and Biomedical Sciences. 2012; 67-78.

[36] Yücel MA, Aasted CM, Petkov MP, Borsook D, Boas DA, Becerra L. Specificity of hemodynamic brain responses to painful stimuli: a functional near-infrared spectroscopy study. Scientific Reports. 2015; 5: 9469.

[37] Akitsuki Y, Decety J. Social context and perceived agency affects empathy for pain: an event-related fMRI investigation. NeuroImage. 2009; 47: 722-734.

[38] Balconi M, Angioletti L. Interoception as a social alarm amplification system. what multimethod (EEG-fNIRS) integrated measures can tell us about interoception and empathy for pain? Neuropsychological Trends. 2021; 29: 39-64.

[39] Bradley M, Lang PJ. Measuring emotion: the self-sssessment manikin and the semantic differential. Journal of Experimental Psychopathology. 1994; 25: 49-59.

[40] Oostenveld R, Praamstra P. The five percent electrode system for high-resolution EEG and ERP measurements. Clinical Neurophysiology. 2001; 112: 713-719.

[41] Jurcak V, Tsuzuki D, Dan I. 10/20, 10/10, and 10/5 systems revisited: their validity as relative head-surface-based positioning systems. NeuroImage. 2007; 34: 1600-1611.

[42] Giacometti P, Perdue KL, Diamond SG. Algorithm to find high density EEG scalp coordinates and analysis of their correspondence to structural and functional regions of the brain. Journal of Neuroscience Methods. 2014; 229: 84-96.

[43] Koessler L, Maillard L, Benhadid A, Vignal JP, Felblinger J, Vespignani $\mathrm{H}$, et al. Automated cortical projection of EEG sensors: Anatomical correlation via the international 10-10 system. NeuroImage. 2009; 46: 64-72.

[44] Pinti P, Scholkmann F, Hamilton A, Burgess P, Tachtsidis I. Current status and issues regarding pre-processing of fNIRS neuroimaging data: an investigation of diverse signal filtering methods within a general linear model framework. Frontiers in Human Neuroscience. 2020; 12: 505.

[45] Pinti P, Aichelburg C, Lind F, Power S, Swingler E, Merla A, et al. Using fiberless, wearable fNIRS to monitor brain activity in realworld cognitive tasks. Journal of Visualized Experiments. 2015; 106: e53336,

[46] Ray RD, Zald DH. Anatomical insights into the interaction of emotion and cognition in the prefrontal cortex. Neuroscience and Biobehavioral Reviews. 2012; 36: 479-501.

[47] Ochsner KN, Gross JJ. The cognitive control of emotion. Trends 
in Cognitive Sciences. 2005; 9: 242-249.

[48] Moriguchi Y, Decety J, Ohnishi T, Maeda M, Mori T, Nemoto K, et al. Empathy and judging other's pain: An fMRI study of alexithymia. Cerebral Cortex. 2007; 17: 2223-2234.

[49] Ochsner KN, Zaki J, Hanelin J, Ludlow DH, Knierim K, Ramachandran $\mathrm{T}$, et al. Your pain or mine? Common and distinct neural systems supporting the perception of pain in self and other. Social Cognitive and Affective Neuroscience. 2008; 3: 144-160.

[50] Balconi M, Canavesio Y. Is empathy necessary to comprehend the emotional faces? The empathic effect on attentional mechanisms (eye movements), cortical correlates (N200 event-related potentials) and facial behaviour (electromyography) in face processing. Cognition and Emotion. 2016; 30: 210-224.

[51] Balconi M, Carrera A. Emotional representation in facial expression and script. A comparison between normal and autistic children. Research In Developmental Disabilities. 2007; 28:409-422.

[52] Balconi M, Canavesio Y. Emotional contagion and trait empathy in prosocial behavior in young people: the contribution of autonomic (facial feedback) and Balanced Emotional Empathy Scale (BEES) measures. Journal of Clinical and Experimental Neuropsychology. 2016; 35: 41-48.

[53] Balconi M, Bortolotti A, Gonzaga L. Emotional face recognition, EMG response, and medial prefrontal activity in empathic behaviour. Neuroscience Research. 2007; 71: 251-259.

[54] Hadjistavropoulos T, Craig KD. A theoretical framework for understanding self-report and observational measures of pain: a communications model. Behaviour Research and Therapy. 2002; 40: 551-570.

[55] Bruce V, Young AW. A theoretical perspective for understanding face recognition. Face and mind (pp. 96-130). Oxford University Press: UK. 1998.

[56] Junghöfer M, Bradley MM, Elbert TR, Lang PJ. Fleeting images: a new look at early emotion discrimination. Psychophysiology. 2001; 38: 175-178.

[57] Pizzagalli D, Koenig T, Regard M, Lehmann D. Affective attitudes to face images associated with intracerebral EEG source location before face viewing. Brain Research. Cognitive Brain Research. 1999; 7: 371-377.

[58] Balconi M, Lucchiari C. In the face of emotions: event-related potentials in supraliminal and subliminal facial expression recognition. Genetic, Social, and General Psychology Monographs. 2005; 131: 41-69.

[59] Balconi M, Lucchiari C. Consciousness and arousal effects on emotional face processing as revealed by brain oscillations. A gamma band analysis. International Journal of Psychophysiology. 2008; 67: 41-46.

[60] Balconi M, Lucchiari C. Event-related potentials related to normal and morphed emotional faces. The Journal of Psychology. 2005; 139: 176-192.

[61] Balconi M, Pozzoli U. Arousal effect on emotional face comprehension. Frequency band changes in different time intervals. Physiology \& Behavior. 2009; 97: 455-462.

[62] Jackson PL, Meltzoff AN, Decety J. How do we perceive the pain of others? A window into the neural processes involved in empathy. NeuroImage. 2005; 24: 771-779.

[63] Jackson PL, Rainville P, Decety J. To what extent do we share the pain of others? Insight from the neural bases of pain empathy. Pain. 2006; 125: 5-9.
[64] Deyo KS, Prkachin KM, Mercer SR. Development of sensitivity to facial expression of pain. Pain. 2004; 107: 16-21.

[65] Prkachin KM, Berzins S, Mercer SR. Encoding and decoding of pain expressions: a judgement study. Pain. 1994; 58: 253-259.

[66] Botvinick M, Jha AP, Bylsma LM, Fabian SA, Solomon PE, Prkachin KM. Viewing facial expressions of pain engages cortical areas involved in the direct experience of pain. NeuroImage. 2005; 25: 312-319.

[67] Saarela MV, Hlushchuk Y, Williams ACDC, Schürmann M, Kalso E, Hari R. The compassionate brain: humans detect intensity of pain from another's face. Cerebral Cortex. 2007; 17: 230-237.

[68] Preston SD, de Waal FBM. Empathy: its ultimate and proximate bases. The Behavioral and Brain Sciences. 2002; 25: 1-71.

[69] Rizzolatti G. The mirror neuron system and its function in humans. Anatomy and Embryology. 2005; 210: 419-421.

[70] Iacoboni M. Imitation, empathy, and mirror neurons. Annual Review of Psychology. 2009; 60: 653-670.

[71] Chakrabarti B, Bullmore E, Baron-Cohen S. Empathizing with basic emotions: common and discrete neural substrates. Social Neuroscience. 2006; 1: 364-384.

[72] Keysers C, Gazzola V. Expanding the mirror: vicarious activity for actions, emotions, and sensations. Current Opinion in Neurobiology. 2009; 19: 666-671.

[73] Ionta S, Costantini M, Ferretti A, Galati G, Romani GL, Aglioti SM. Visual similarity and psychological closeness are neurally dissociable in the brain response to vicarious pain. Cortex. 2020; 133 : 295-308.

[74] López-Solà M, Koban L, Krishnan A, Wager TD. When pain really matters: a vicarious-pain brain marker tracks empathy for pain in the romantic partner. Neuropsychologia. 2020; 145: 106427.

[75] Farb NAS, Segal ZV, Mayberg H, Bean J, McKeon D, Fatima Z, et al. Attending to the present: mindfulness meditation reveals distinct neural modes of self-reference. Social Cognitive and Affective Neuroscience. 2007; 2: 313-322.

[76] Farb NAS, Anderson AK, Mayberg H, Bean J, McKeon D, Segal ZV. Minding one's emotions: mindfulness training alters the neural expression of sadness. Emotion. 2010; 10: 25-33.

[77] Xie J, Yang H, Xia X, Yu S. The influence of medical professional knowledge on empathy for pain: Evidence from fNIRS. Frontiers in Psychology. 2018; 9: 1-8.

[78] Heinzel S, Haeussinger FB, Hahn T, Ehlis A, Plichta MM, Fallgatter AJ. Variability of (functional) hemodynamics as measured with simultaneous fNIRS and fMRI during intertemporal choice. NeuroImage. 2013; 71: 125-134.

[79] Schroeter ML, Bücheler MM, Müller K, Uludağ K, Obrig H, Lohmann $\mathrm{G}$, et al. Towards a standard analysis for functional nearinfrared imaging. NeuroImage. 2004; 21: 283-290.

[80] Wobst P, Wenzel R, Kohl M, Obrig H, Villringer A. Linear aspects of changes in deoxygenated hemoglobin concentration and cytochrome oxidase oxidation during brain activation. NeuroImage. 2001 ; 13: 520-530.

[81] Villringer A, Dirnagl U. Coupling of brain activity and cerebral blood flow: basis of functional neuroimaging. Cerebrovascular and Brain Metabolism Reviews. 1995; 7: 240-276.

[82] Schroeter ML, Kupka T, Mildner T, Uludağ K, Von Cramon DY. Investigating the post-stimulus undershoot of the BOLD signal -a simultaneous fMRI and fNIRS study. Neuroimage. 2006; 30: 349_ 358. 